\title{
Effect of prone sleeping on circulatory control in infants
}

\author{
Angeline Chong, Nuala Murphy, Thomas Matthews
}

\begin{abstract}
Background-The mechanism of death in sudden infant death syndrome (SIDS) remains unclear. Progressive bradycardia is the pre-eminent terminal event, suggesting that circulatory failure might be a crucial factor. Vasomotor tone regulates the circulatory system by controlling blood volume distribution while maintaining venous return and blood pressure.

Aim-To examine whether prone sleeping, the most consistently identified risk factor for SIDS, has a measurable influence on vasomotor/circulatory control.

Methods-44 full term infants (mean age, 7.9 weeks) were studied during an overnight sleep. Recordings were made while the infants were horizontal and asleep in the supine and prone positions, and repeated after a head up tilt to $60^{\circ}$, maintained for $\mathbf{3 0}$ minutes, while in both sleep positions. Blood pressure, heart rate, anterior shin, and anterior abdominal wall skin temperatures were measured.

Results-Systolic blood pressure was lower, but peripheral skin temperature and heart rate were higher during sleep, while horizontal, in the prone rather than the supine position. After tilting, there was a greater reduction in blood pressure and a greater increase in peripheral skin temperature and heart rate when in the prone position. Anterior abdominal wall skin temperature did not vary in either sleeping positions while horizontal or tilted.
\end{abstract}

Conclusion-Prone sleeping has a measurable effect on circulatory control, with a reduction in vasomotor tone resulting in peripheral vasodilatation, a higher peripheral skin temperature, a lower blood pressure, and a higher resting heart rate. Because vasomotor tone is crucially important in circulatory control this could be a factor in increasing the risk of SIDS. (Arch Dis Child 2000;82:253-256)

Keywords: sudden infant death syndrome; autonomic function; head up tilt test; vasomotor tone

Department of Paediatrics, Rotunda Hospital, Dublin 1, Republic of Ireland

A Chong

N Murphy

T Matthews

Correspondence to:

Professor T Matthews

Accepted 14 October 1999 airway patency control, transcutaneous oxygen saturation, the frequency of gastro-oesophageal reflux, or rectal temperature control in

Recent epidemiological studies have heating increase the risk of sudden infant death syndrome (SIDS), although the mechanism is unknown. ${ }^{12}$ Prone sleeping has been shown not to affect adversely respiratory control, infants. ${ }^{3-6}$ A report of infants dying from SIDS while attached to a cardiorespiratory monitor, with a built in memory, showed a progressive bradycardia with continued breathing movements to be the most frequent terminal event. ${ }^{7}$ Analysis of the data from these deaths suggests that cardiovascular/circulatory failure is important in the sequence of events leading up to SIDS. The vasomotor system plays an important role in circulatory control by regulating and altering blood volume distribution to meet the needs of different organs and tissues while maintaining a normal blood pressure, an adequate central venous return and cardiac output. The aim of our study was to determine whether sleeping in the prone position has a measurable effect on vasomotor/circulatory control in healthy infants.

\section{Patients and methods}

Full term, healthy infants were recruited from the postnatal ward at the Rotunda Hospital (Dublin, Republic of Ireland) (between days 1 to 5 of life). Of the 88 infants that were initially recruited, 61 returned to be studied. No infant was on medication and they were admitted electively into the paediatric unit and studied during unsedated overnight sleep (between the hours of 22:00 and 02:00). Because of the difficulty in keeping some of the infants asleep for the duration of the study period, complete data are available for only 44 of the infants. There were 21 boys and 23 girls, and the mean age at study was 7.9 weeks (range, 6-9 weeks). Seventeen of the babies were breast fed, whereas the others were fed formula milk. After being fed, infants were put to sleep in a plastic bassinet on a mechanically controlled tilt table in the supine position, wearing a vest, a "babygro", and a cardigan, with two small single blankets (tog 10). Resting measurements of heart rate, blood pressure, anterior shin temperature, and anterior abdominal skin temperature were recorded for a minimum of 30 minutes of sleep time. The infants were then tilted head up to $60^{\circ}$ over a period of three to four seconds and the tilt was maintained for 30 minutes. Measurements of heart rate, blood pressure, and anterior shin and abdominal skin temperatures were made for another 30 minutes, after which time the infant was returned to the horizontal position and the sleep position was changed to prone.

Physiological parameters were measured for a minimum of 30 minutes in the prone position, then the infants were again tilted head up to $60^{\circ}$ and recordings were made for a further 30 minutes. All recordings were made during clinically staged quiet sleep (defined as: 
eyes closed with no body or eye movements, and the cardiorespiratory tracing showing respiratory movements of uniform amplitude). ${ }^{8}$ During periods of active sleep, recordings were discontinued and only restarted when quiet sleep was established again. Therefore, all recordings were made during quiet sleep. The room temperature was maintained at $23-25^{\circ} \mathrm{C}$. Informed parental consent was obtained in all cases and no financial incentives were given to the families for participating in our study. Approval was given for our study by the hospital ethics committee.

\section{MEASUREMENTS OF PHYSIOLOGICAL PARAMETERS}

Heart rate

Continuous electrocardiographic (ECG) recordings, and respiratory and abdominal wall movements, were recorded on a Corometrics 552 neonatal cardiorespiratory monitor (Corometrics Medical Systems, Connecticut, USA) and stored on paper at a speed of $6.25 \mathrm{~mm} / \mathrm{s}$. The heart rate/min was then assessed by counting the number of $\mathrm{R}$ waves over a one minute period. The mean heart rates over 30 minutes while the infants were horizontal and tilted to $60^{\circ}$ were used to compare the prone with the supine position.

\section{Blood pressure}

Blood pressure was measured by a neonatal blood pressure monitor (Spacelabs NIBP monitor; Spacelabs, Redmond, West Virginia, USA) using an appropriately sized cuff attached to the right upper arm. The mean blood pressure measurements of readings taken at five minute intervals for 30 minutes while the infants were in the horizontal position were used to compare the supine with the prone position. After tilting, readings were then taken one minute and five minutes after tilting and at five minute intervals for a total of 30 minutes. Differences between the mean blood pressure readings over the 30 minute period after tilting and the resting blood pressure, which was defined as the mean of five readings taken at one minute intervals immediately before tilting, were taken as a response to tilting. Systolic blood pressure was used to express the results.

\section{Skin temperature}

Skin temperature was measured by placing and taping a thermistor probe on the right anterior shin and a second probe on the left anterior abdominal wall. These were recorded continuously at one minute intervals by a Squirrel 1000 datalogger (Grant Instruments, Cambridge, UK) for a minimum of 30 minutes in each sleep position (supine, tilted in the supine position, prone, and tilted in the prone

Table 1 Physiological parameters during 30 minutes of supine and prone sleep in the horizontal position

\begin{tabular}{lrrl}
\hline & \multicolumn{1}{l}{ Supine } & Prone & p Value \\
\hline Heart rate $(\mathrm{bpm})$ & $130.8(7.9)$ & $131.4(7.5)$ & NS \\
Systolic blood pressure $(\mathrm{mm} \mathrm{Hg})$ & $82.3(8.8)$ & $79.6(8.9)$ & $<0.05$ \\
Peripheral skin temperature $\left({ }^{\circ} \mathrm{C}\right)$ & $33.3(1.4)$ & $33.7(1.4)$ & $<0.05$ \\
Anterior abdominal skin temperature $\left({ }^{\circ} \mathrm{C}\right)$ & $35.7(0.4)$ & $35.8(0.5)$ & NS
\end{tabular}

Values are means $(\mathrm{SD})$

bpm, beats/minute.
Table 2 The responses of the physiological parameters during 30 minutes' head up tilt in the supine and prone sleeping positions

\begin{tabular}{lll}
\hline & Supine & Prone \\
\hline Heart rate $(\mathrm{bpm})$ & 129.7 & $132.9^{\star}$ \\
Systolic blood pressure $(\mathrm{mm} \mathrm{Hg})$ & $-1.8 \%$ & $-3 . \%^{\star}$ \\
Peripheral skin temperature $\left({ }^{\circ} \mathrm{C}\right)$ & +0.1 & $+0.3^{\star}$ \\
Anterior abdominal skin temperature $\left({ }^{\circ} \mathrm{C}\right)$ & -0.1 & +0.1 \\
\hline
\end{tabular}

${ }^{\star} \mathrm{p}<0.05$

bpm, beats/minute.

position). Ambient temperature was also recorded. Comparisons were made using the mean temperature measurements over each 30 minute period.

ANALYSIS

Statistical comparisons were made by the Student's $t$ test.

\section{Results}

Systolic blood pressure was lower and peripheral skin temperature was higher in the prone than in the supine position while horizontal (table 1). The resting heart rate was slightly, although not significantly, higher in the prone position. In response to head up tilting to $60^{\circ}$, the infants developed a greater reduction in blood pressure and a greater increase in peripheral skin temperature and heart rate when in the prone position compared with the supine position (table 2). Anterior abdominal skin temperature did not vary with either position or head up tilting.

\section{Discussion}

The risk of SIDS increases dramatically in the 2nd and 3rd months of postnatal life, with most deaths occurring during the overnight sleep period. ${ }^{9}$ Because circulatory control seems to be important in the sequence of events leading to death in SIDS the effect of sleep, especially overnight in the prone position, on circulatory control might provide some insight into the mechanism of death. Our results suggest that sleep in the prone position is associated with a reduction in vasomotor tone, with a lower resting blood pressure $(79.6 \mathrm{~mm} \mathrm{Hg} v 82.3 \mathrm{~mm}$ $\mathrm{Hg}$ ) and a higher peripheral skin temperature $\left(33.7^{\circ} \mathrm{C} v 33.3^{\circ} \mathrm{C}\right)$ in the prone position compared with the supine sleeping position. The lack of an effect of the prone or the supine sleeping position on anterior abdominal skin temperature $\left(35.8^{\circ} \mathrm{C} v 35.7^{\circ} \mathrm{C}\right)$ makes it unlikely that lying on the probe, while prone, influenced the results. In addition, when circulatory control was stressed, by a $60^{\circ}$ head up tilt for 30 minutes, the fall in resting blood pressure was greater while asleep prone than supine, and was matched by a greater increase in peripheral skin temperature, and an increased resting heart rate (table 2), indicating peripheral pooling of blood. These results are supported by two previous studies of peripheral skin temperature in similar aged infants during an overnight sleep. Skadberg and Markestad studied 32 infants aged 2.5 and 5 months and found that prone sleeping was associated with a higher peripheral (toe) skin temperature and a faster resting heart rate when compared with supine sleeping. ${ }^{10}$ The peripheral 
skin temperatures in this study were higher than in our study $\left(36.3^{\circ} \mathrm{C} v 33.7^{\circ} \mathrm{C}\right)$; however, their infants were more heavily wrapped (15.5 togs $v$ 10 togs) in a similar ambient temperature. The amount of clothing and bedding used in both studies is within the range normally used at home at this age. ${ }^{11}$ Skadberg and Markestad concluded that the tachycardia associated with prone sleeping, seen in their study, was the result of heat stress, and occurred despite infants in the prone position having more settled sleep with less arousals, confirming the findings of previous studies that infants sleep "better" if prone (prone sleeping was previously recommended for this reason). ${ }^{12-14}$

These two studies give some insight into the circulatory effects of two differing, but normal, infant care practices, with heavier wrapping causing a higher peripheral skin temperature and a faster resting heart rate. Heavy wrapping, especially if sleeping prone, has been a consistent risk factor for SIDS, ${ }^{15}$ and a faster resting heart rate has been one of the most consistent factors found in cardiorespiratory recordings of infants subsequently dying of SIDS. ${ }^{16}{ }^{17}$ Azaz et al studied 22 infants in the prone position during an overnight sleep in conditions very similar to our study and found similar anterior shin skin temperatures $\left(33.7^{\circ} \mathrm{C} v 33.7^{\circ} \mathrm{C}\right) .^{18} \mathrm{Azaz}$ et al also found that the anterior shin skin temperature during sleep increased significantly from 1-12 weeks of age, as does the resting heart rate, ${ }^{19}$ suggesting an age associated reduction in vasomotor tone during sleep, with peripheral vasodilatation, a reduction in venous return, and a compensatory increase in resting heart rate. In our study, all infants were studied in the supine sleeping position first, because there were no regular prone sleepers in the group, and consequently the differences found between prone and supine sleep could reflect the fact that all the prone studies took place later during the night's sleep. However the peripheral skin temperature differences between prone and supine sleep in our study are very similar to those found in the study of Skadberg et al, where infants were allocated randomly to the prone or supine position initially, and where peripheral skin temperature was higher when prone both in active and quiet sleep at 2.5 and 5 months, whether studied early or late in an overnight sleep. Consequently, we feel that the differences shown in our study reflect an effect of sleep in the prone position.

The most consistent circulatory effect of sleep is a reduction in vasomotor tone, reflected by a fall in resting blood pressure and a decrease in peripheral vascular resistance. ${ }^{20}$ This is associated with a reduced heart rate, a reduced central venous return, and a reduction in cardiac output, indicating a dramatic sleep induced alteration in baroreceptor function, because a similar combination of events while awake is associated with an increase in heart rate, with sympathetic activation and peripheral vasoconstriction. ${ }^{20}$ Overheating and overwrapping increase the risk of SIDS, especially if sleeping prone, ${ }^{12}$ and the normal response to heat is to increase skin blood flow and heat loss by a reduction in vasomotor tone and peripheral vasodilatation. ${ }^{21}$

Bundling (or tightly wrapping infants) has also been shown to increase the risk of SIDS in infants sleeping prone and also to increase skin temperature, implying peripheral vasodilatation. ${ }^{12} 22$ In addition, co-sleeping has been reported to increase the risk of SIDS and is associated with a higher rectal temperature in infants, again implying a degree of heat stress. ${ }^{23-25}$ Sleep is also associated with a fall in rectal temperature, which is absent during the first 2 weeks of postnatal life, a period of low risk of SIDS, and which becomes established and increases in magnitude by 8 weeks, a period of increased risk of SIDS. ${ }^{26}$ This sleep induced fall in rectal temperature is associated with a maintained or rising peripheral skin temperature, which often exceeds rectal temperature, implying both peripheral vasodilatation and age associated changes in vasomotor control during sleep. ${ }^{27}$ Vasomotor control has long been known to be poor in healthy full term infants, with a $15 \%$ reduction in circulating blood volume resulting in a $54 \%$ reduction in central venous return because of poor peripheral vasoconstrictor ability. ${ }^{28}$

The vasomotor system is controlled by the sympathetic component of the autonomic nervous system, which also regulates cardiac repolarisation. Recently, a prolonged QT interval has been shown to increase the risk of SIDS and inefficient cardiac repolarisation has been suggested to be the cause. ${ }^{29}$ We have suggested previously that poor autonomic control, in particular the combination of a sleep induced reduction in vasomotor tone with a reduction in central venous return and cardiac distension, might trigger a brain stem mediated bradycardia in some infants via a neurocardiac reflex. ${ }^{30}{ }^{31}$ However, without invoking a specific neurocardiac reflex, a combination of circumstances, each of which reduce vasomotor tone, such as nocturnal sleep in the prone position at 2 months' postnatal age in a heavily wrapped infant, associated with an unresponsive baroreceptor controlling system, could render an infant vulnerable to inadequate central venous return, poor pulmonary perfusion, and hypoxia. Antemortem pulmonary thrombi have been described in SIDS, suggesting low pulmonary blood flow in some instances. ${ }^{32}$ Prone sleeping might also impair the arousal response, perhaps because the infants are more deeply asleep, further disadvantaging infants with a down regulated baroreceptor response. ${ }^{33}$ The peak age of occurrence of SIDS coincides with the nadir of the physiological anaemia that occurs in all infants and which would accentuate any sleep induced reduction in venous return. Physiological anaemia is exaggerated in preterm and low birth weight infants, who also have an increased risk of SIDS. ${ }^{9}$ Our study suggests that the normal sleep induced reduction in vasomotor tone is greater in the prone sleeping position, perhaps simply because in this position infants are more deeply asleep, and that vasomotor control and its interaction with circulatory control and temperature control, deserve further study in infants. 
We thank the following for financial support: Foundation for the study of Infant Deaths, Friends of the Rotunda Hospital, Temstudy of Infant Deaths, Friend
ple Street Children's Hospital.

1 Fleming PJ, Gilbert RE, Azaz Y, et al. The interaction between bedding and sleeping position in sudden infant between bedding and sleeping position in sudden infant death syndrome: a p

2 Ponsonby AL, Dwyer T, Gibbons LE, et al. Thermal environment and sudden infant death syndrome: case control study. BMF 1992;304:277-82.

3 Orr WC, Monte LS, Duke MS, et al. Effect of sleep state and position on the incidence of obstructive and central apnoea in infants. Pediatrics 1985;75:832-5.

4 Levene S, McKenzie SA. Transcutaneous oxygen saturation in sleeping infants: prone and supine. Arch Dis Child 1990; 65:524-6.

5 Orenstein SR, Whitington PF. Positioning for the prevention of infant gastroesophogeal reflux. F Pediatr 1983;103: 534-7.

6 Petersen SA, Anderson ES, Lodemore M, Rawson D, Wailoo MP. Sleeping position and rectal temperature. Arch Dis Child 1991;66:976-9.

7 Meny RG, Carroll JL, Carbone MT, Kelly DH. Cardiorespiratory recordings from infants dying suddenly and respiratory recordings from infants dying sudde

8 Tappin DM, Ford RPK, Nelson KP, et al. Breathing, sleep state, and rectal temperature oscillations. Arch Dis Child $1996 ; 74: 427-31$

9 Gibson AAM. Current epidemiology of SIDS. $f$ Clin Pathol 1992;45(suppl): $7-10$

10 Skadberg BT, Markestad T. Behavior and physiological responses during prone and supine sleep in early infancy. Arch Dis Child 1997;76:320-4.

11 Wailoo MP, Petersen SA, Whittaker H, Goodenough P. The thermal environment in which 3-4 month old infants sleep at home. Arch Dis Child 1989;64:600-4.

12 Douthitt TC, Brackbill Y. Differences in sleep, waking and motor activity as a function of prone or supine resting position in the human neonate. Psychophysiology 1972;9:99100.

13 Kahn A, Grosswasser J, Sottiaux M, Rebuffat E, Franco E, Dramaix M. Prone or supine position and sleep characteristics in infants. Pediatrics 1993;6:1112-15.

14 Jolly H. Book of child care. London: Sphere, 1977.

5 Ponsonby AL, Dwyer T, Gibbons LE, Cochrane JA, Wang TG. Factors potentiating the risk of sudden infant death syndrome associated with the prone position. $N$ Eng $\mathcal{F} M e d$ 1993;339:377-82.

16 Wilson AJ, Stevens V, Franks CI, Southall DP. Analysis of long-term recordings from infants who subsequently suffered SIDS. The sudden infant death syndrome cardiac and respiratory mechanisms and interventions. Ann NY Acad Sci 1988;533:390-410.
17 Kelly DH, Golub H, Carley C, Shannon DC. Pneumograms in infants who subsequently died of sudden infant death syndrome. F Pediatr 1986;109:249-54.

18 Azaz Y, Fleming PJ, Levene M, McCabe R, Stewart A, Johnson P. The relationship between environmental temperature, metabolic rate, sleep state, and evaporative water loss in infants from birth to three months. Pediatr Res 1992;32:417-23.

19 Lipton EL, Steinschneider A, Richmond JB. The autonomic nervous system in early life. $N$ Engl f Med 1965;273:14753.

20 Mancia G. Autonomic modulation of the cardiovascular system during sleep. $N$ Engl f Med 1993;339:347-9.

21 Rutter N, Hull D. Response of term babies to a warm environment. Arch Dis Child 1979;54:178-83.

22 Grover G, Berkowitz CD, Lewis RJ, Thompson M, Berry L, Seidel J. The effects of bundling on infant temperature. Pediatrics 1994;94:669-73.

23 Brooke H, Gibson A, Tappin D, Brown H. Case control study of sudden infant death syndrome in Scotland, 1992-5. BMF 1997;314:1516-20.

24 Fleming PJ, Blair PS, Bacon C, et al. Environment of infants during sleep and risk of the sudden infant death syndrome: results of 1993-95 case-control study for confidential inquiry into stillbirths and deaths in infancy. $B M F$ 1996;313:191-5

25 Tuffnell CS, Petersen SA, Wailoo MP. Higher rectal temperatures in co-sleeping infants. Arch Dis Child 1996;75:249-50.

26 Lodemore MR, Petersen SA, Wailoo MP. Factors affecting the development of night time temperature rhythms. Arch Dis Child 1992;67:1259-61.

27 Wailoo MP, Petersen SA, Whittaker H, Goodenough P. Sleeping body temperature in 3-4 month old infants. Arch Dis Child 1989;64:596-9.

28 Wallgren G, Hanson JS, Lind J. Quantitative studies of the human neonatal circulation. Acta Paediatr Scand 1967; 9(suppl):43-68.

29 Schwartz PJ, Stramba-Badiale M, Segantini A, et al. Prolongation of the Q-T interval and the sudden infant death syndrome. N Engl f Med 1998;338:1709-14.

30 Matthews TG. The autonomic nervous system - a role in sudden infant death syndrome. Arch Dis Child 1992;67: 654-6.

31 Ledwidge M, Fox GPP, Matthews TG. Neurocardiogenic syncope: a model for SIDS. Arch Dis Child 1998;78:481-3.

32 Barrett AM. Sudden death in infancy. Recent advances in paediatrics. London: JA Churchill, 1954:301-20.

33 Galland BC, Reeves G, Taylor BJ, Bolton DPG. Sleep position, autonomic function, and arousal. Arch Dis Child 1998;78:189-94. 